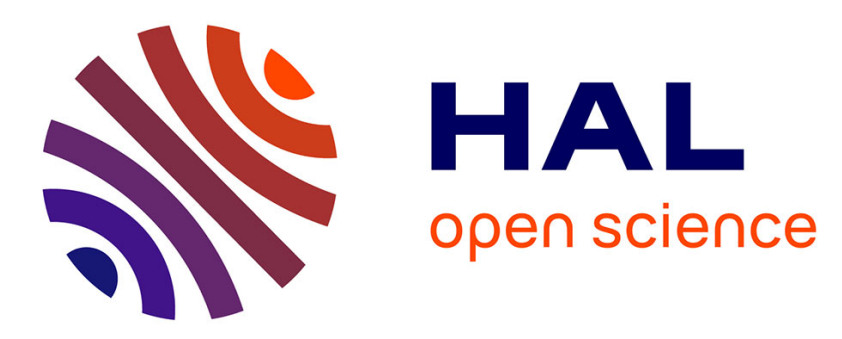

\title{
Evidence, origin and impact of liquid flows in plasma medicine in vitro treatments with APPJs
}

\author{
Augusto Stancampiano, Pablo Escot Bocanegra, Sébastien Dozias, \\ Jean-Michel Pouvesle, Robert Eric
}

\section{To cite this version:}

Augusto Stancampiano, Pablo Escot Bocanegra, Sébastien Dozias, Jean-Michel Pouvesle, Robert Eric. Evidence, origin and impact of liquid flows in plasma medicine in vitro treatments with APPJs. Plasma Sources Science and Technology, 2021, 30 (1), pp.015002. 10.1088/1361-6595/abcc50 . hal-03360207

\section{HAL Id: hal-03360207 https://hal.science/hal-03360207}

Submitted on 30 Sep 2021

HAL is a multi-disciplinary open access archive for the deposit and dissemination of scientific research documents, whether they are published or not. The documents may come from teaching and research institutions in France or abroad, or from public or private research centers.
L'archive ouverte pluridisciplinaire $\mathbf{H A L}$, est destinée au dépôt et à la diffusion de documents scientifiques de niveau recherche, publiés ou non, émanant des établissements d'enseignement et de recherche français ou étrangers, des laboratoires publics ou privés. 


\title{
Evidence, origin and impact of liquid flows in plasma medicine in vitro treatments with APPJs
}

\author{
Augusto Stancampiano $\odot$, Pablo Escot Bocanegra, Sébastien Dozias, \\ Jean-Michel Pouveslę and Eric Robert $\odot$
}

GREMI, UMR7344 CNRS/Université d'Orléans, 14 rue d'Issoudun, 45067 Orléans, France

E-mail: augusto.stancampiano@univ-orleans.fr

Received 7 August 2020, revised 3 November 2020

Accepted for publication 19 November 2020

Publisbed XX XX XXXX

\begin{abstract}
Plasma jets are being intensively studied for biomedicine applications but their fine control remains challenging due to the mutual interactions between plasma and target. Even considering perhaps one of the simplest scenario in a research laboratory, using a plasma jet to treat a 2D cells culture in a plastic multi-well plate, it is not known in detail how the physical environment of the micro-well may influence the nature of the plasma jet treatment. This study aims to shed light by investigating for the first time the liquid flows induced by the plasma treating a biologically-relevant liquid (e.g. PBS) in standard tissue culture grade multi well plates (i.e. 96, 24, 12 and 6 wells). Evaporation, induced by the anhydrous gas flow, is demonstrated to govern the thermal effect and be at the origin of the observed liquid cooling. Moreover, the formation of stable vortexes in the liquid induce, a non-uniform distribution of plasma generated reactive species. This phenomenon, reported for various vessels and volumes of liquid, does not originate from thermal gradients but is instead the likely result of a combination of electrohydrodynamic forces and gas flow tangential components induced by the plasma impinging on the liquid surface.
\end{abstract}

Keywords: electrohydrodynamic, atmospheric pressure plasma, liquid target, Schlieren, evaporation, plasma medicine, vortex recirculation

(Some figures may appear in colour only in the online journal)

\section{Introduction}

Atmospheric pressure plasma jets (APPJ) are nowadays maybe the most versatile, widely adopted and investigated type of atmospheric plasma reactor for a great variety of biomedical and material processing innovative applications.

In the last decades the scientific effort on APPJs has gradually shifted from their investigation in free flow conditions to the study of their interaction with different targets [1]. The relation between plasma and target has been demonstrated to be mutual, potentially affecting both the target and the plasma characteristics [2-4]. The interaction between plasma and liquids has been the subject of intense research, in particular

- Author to whom any correspondence should be addressed. focused on the production of bio-active reactive oxygen and nitrogen sperias (RONS) in placms rmated licuids This mpic in fact is of great relevance for plasma biomedical applications where precise control over these reactive species is mandatory to achieve the desired biological response.

Concerning targets in plasma biomedical studies, there is a certain uniformity in commonly adopted in vitro configurations due to the standardized vessels (e.g. tissue culture grade multi well plate, Petri dishes) and liquids (e.g. PBS and culture media) routinely used for microbiology assays.

The characteristics of these targets and setups certainly influence the plasma treatment conditions but in spite of their large diffusion, they have been very scarcely investigated from a physical point of view. Only few papers reported on these configurations rising awareness on the influence of seldom 
controlled parameters such as the in vitro target support or the vessel border height $[2,5,6]$. Such parameters can significantly alter the RONS produced by the plasma and their distribution at the target surface and bulk. It is reasonable to suspect that other features and parameters not yet investigated may play a similar role in governing plasma biomedical treatment.

Thus, it appears critical and relevant to undergo a physical investigation of the adopted setups even when the main focus of the research maybe purely biological. For example, in the plasma medicine context the understanding of plasmaliquid interaction is of major importance since water is the main component of most targets (e.g. culture medium, tissues, ...,) [7].

As a representative example, Sasaki et al [8] reported on how the plasma-induced cell membrane permeabilisation decreased markedly with increasing liquid volume covering the cell culture. This is believed to be linked to the increased distance between liquid surface and cells, which prevents short-species such as $\mathrm{OH}$ to reach the bottom of the well due to their short diffusion length in the order of few hundred micrometers. The addition of few milliliters of culture medium while harmless from a purely biological point of view could completely change the plasma treatment outcome. The transport of reactive species and its limits control in many cases the plasma treatment efficiency and outcome [9].

Similarly, studies non related to the plasma domain reported on how natural convection can take place inside Petri dishes in incubators and lead to non-uniform cell culture [10].

Riès et al [4] reported on how the chemical composition (especially water related species) of a plasma jet can be significantly affected by the target composition and electrical characteristics. Always in the case of an APPJ impinging on a liquid Kondeti et al [11] observed higher decomposition rate of crystal violet for oscillating surface dimple and suspected a possible role of vortex recirculation. The dependency of interfacial dynamic (in that case the dimple oscillation) and the vessel dimension was also highlighted in the work.

Awareness is rising that only a combined characterization of both the treatment conditions and effects could provide the necessary insight on the leading mechanisms of plasma action.

In this context, some papers already reported on how an APPJ impinging on a liquid surface is likely to induce modifications of the fluid dynamic regime in the bulk liquid. Two main effects have been observed: a fast liquid flow moving away from the impinging point; the formation of stable vortexes with velocities up to several centimeters per second.

In the first case, it has been reported on how the impinging of a plasma jet on a liquid can induce a fast linear flow going in the depth direction within the first seconds $(<10 \mathrm{~s})$ from the plasma ignition. The group of Kawasaki in particular investigatred this rfferst in enrrelation with the fast transport of ROS in the bulk liquid and the supply of these species to the bottom of the vessel [12-14]. The fast liquid flow appears visible as rich of ROS that react with the dissolved KI-starch leading to a colour variation. Rumbach et al [15] also reported a similar fast flow taking place in the first $5 \mathrm{~s}$ of plasma treatment, for a different sefup and using $\mathrm{pH}$ sensitive reagents In this case a basic $\mathrm{pH}$ was associated with the observed fast flow. It was hypothesized that charged species accumulation and EHD forces were the main reasons for measured effect on $\mathrm{pH}$ modulation.

Mitsugi et al [16] reported on the possible role of plasmainduced pressure waves as a possible cause of this ROS fast flow but only for small gas flow rate ( $<1 \mathrm{slm})$ as for higher flow the stream was not detected although the intensity of pressure waves increased. Always the same group observed the same phenomenon through PIV technique confirming the short duration of this fast flow.

As suggested by Kawasaki et al [17] this fast linear flow going in the depth of the vessel is inversely dependent on the gas flow rate and the gap distance between the plasma reactor and the liquid surface. In their work, they assumed the possible role of vortex recirculation, that could take place inside the liquid, in opposing the linear fast flow so blocking it for certain conditions [14].

Dealing with the second class of plasma induced effect in liquid samples, vortex recirculation has indeed been reported hy other studies on APPI trestment of a liquid volume Kovacevic et al [18] reported on the Schlieren visualization of these vortex formations and the reversing of the rotation direction when plasma is ignited compared to gas only conditions. Thagard et al observed vortex formations as well in considerably larger vessels (several cm side) for a rod-plane configuration without gas flow [19]. Conversely, these formations have been marginally reported in other paperfor different plasma jet sources [20-24].

Due to the relatively slow diffusion of reactive species in the liquid phase, the mixing caused by this fluid dynamic plays a critical role in the transport and chemical balance following plasma jet action on liquid samples [20]. Thus, the study of the plasma-induced liquid fluid dynamic and its origins appears as a critical issue to be investigated in detail to achieve proper control over plasma and liquid processes. Concerning the vortex recirculation various explanations (figure 1) for the causes at the origin of these phenomena have been advanced:

(a) Buoyancy forces and Marangoni cffect

(b) Gas flow shear stress

(c) Electrohydrodynamic (EHD) forces

Usually only one or two of these causes are taken into account at a time in the literature. Moreover, most of the pioneering papers previously mentioned address the subject marginally $[18,21,25]$ and usually investigate setups with custom vessels containing large volume of liquid (tens or hundreds of millilitres) $[19,21]$, usually distilled water.

Prompted by the potentially huge impact of this plasma indures vortex recirrulation and the possible dependency between vessel size and interfacial dynamic, in this work, the effect is investigated in small and very small vessels typical of the plasma medicine experimental setups. Furthermore, the up today's suspected causes of this phenomenon are addressed systematically from an experimental and analytical point of view so to infer general considerations and guidelines that could be applied to other similar setups. 


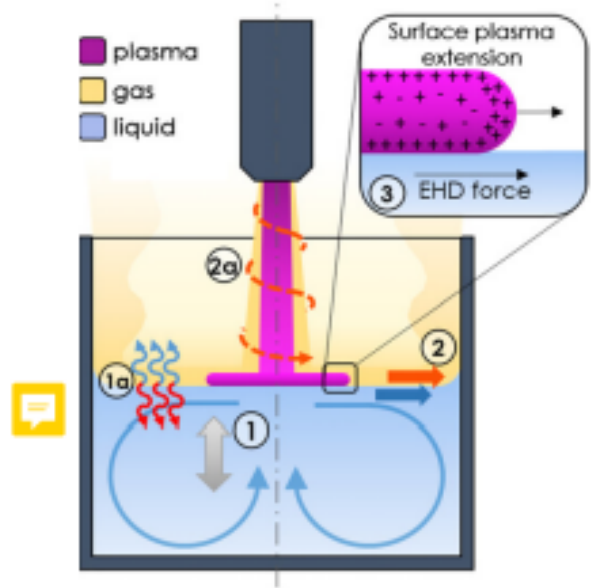

Figure 1. Conceptual representation of the forces and effects taking place when an APPJ impinges on a liquid contained in a vessel: (1) Buoyancy forces and Marangoni effect possibly caused by concentration gradients or (1a) heat transfer; (2) gas flow shear stress on the liquid surface potentially affected by (2a) plasma-induced gas flow modification; (3) EHD forces.

In its final intent, the work aims not only at bringing new insight on plasma induced liquid flows but also at rising awareness on their impact in widely adopted experimental setups such as those encountered in plasma medicine in-vitre experiments.

\section{Methodology}

The atmospheric plasma jet used in this work is a plasma gun (PG) already described in detail in [26]. Briefly, the PG is a coaxial DBD reactor with a quartz capillary flushed with helium and powered by a micropulsed high voltage generator. The $12 \mathrm{~cm}$ long capillary was tapered at the outlet $\left(\emptyset_{\text {in }}=1.3 \mathrm{~mm}, \emptyset_{\text {ext }}=3 \mathrm{~mm}\right)$. The source was flushed with a helium flow ( 1 or $2 \mathrm{slm}, 99999 \%$ pure, water content $<0.5 \mathrm{ppm}$ ) and powered by $2 \mu$ s (FWHM) duration voltage pulses of $12 \mathrm{kV}$ peak that could be set to be either with positive or negative polarity. The repetition rate of the pulses was fixed at $1 \mathrm{kHz}$. The plasma source was positioned vertically over the sample (figure 2). The discharge gap (distance between the nozzle and the surface of the liquid) was fixed at 5 and $15 \mathrm{~mm}$.

The adopted vessels were 96, 24 and 12-multiwell microplates (Nunclon ${ }^{8}$ Delta Surface, Thermo Fisher Scientific, DK), 6-multiwell plates (TPP flat bottom, SigmaAldrich, FR) and a glass test tube (Thermo Fisher Scientific, DK).

Moreover a custom-made 3D-printed vessel with two paralIel glass windows was created specifically for this investigation (figure 3). The vessel was designed to present a cubic cavity of $16 \times 16 \times 16 \mathrm{~mm}^{3}$.

These dimensions were chosen in order to reproduce as close as possible those of the 24-multiwell microplate (cylinder $\emptyset 16 \mathrm{~mm}, \mathrm{H}: 16 \mathrm{~mm}$ ) while having parallel walls so to be more convenient for Schlieren visualization.

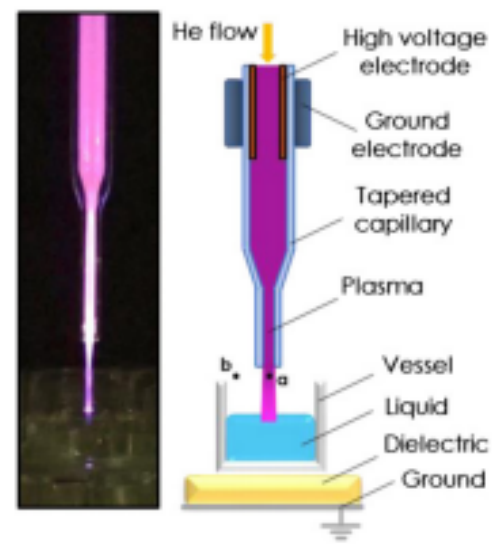

Figure 2. Photo and schematic representation of the experimental setup. The points ' $a$ ' and ' $b$ ' are the position of the optical probe for gas temperature measurements. $1 \mathrm{~mm}$ away from the capillary orifice and either on the jet axis (a) or $5 \mathrm{~mm}$ radially away from it (b).

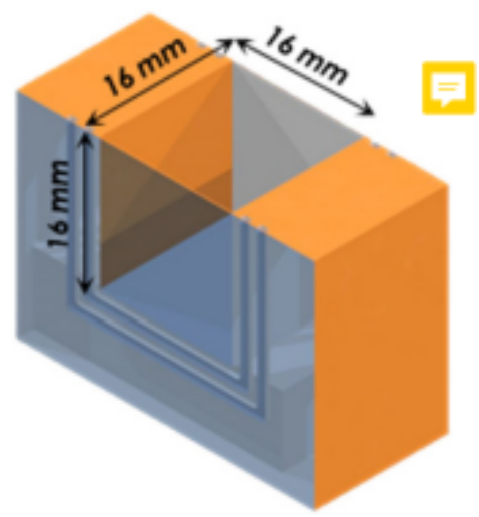

Figure 3. Representation of the custom square-base vessel adopted for Schlieren investigation. The characteristic length scale is the same as that of a standard round-based well of a 24-multiwell microplate.

Even if with different geometry (cubic instead of cylindrical) the custom container has the same characteristic length scale of a standard well adopted for in vitro experiments and should therefore present comparable fluid dynamic regimes. The relevance of this cubic vessel to mimic liquid dynamics in cylindrical cell culture wells will be further detailed along the manuscript.

During treatment the vessels where positioned on a $1 \mathrm{~cm}$ thick dielectric PVC plate positioned on a grounded table. The relatively big impedance of the support allows to neglect the small differences in wall thickness and materials properties between the various vessels.

The liquid adopted for the test was PBS (Dulbecco's phosphate buffered saline by Gibco, $90 \%$ water). A total amount of $200 \mu \mathrm{l}, 3 \mathrm{ml}$ and $4 \mathrm{ml}$ was used in the 96,12 and 6 -well plates respectively. The test tube was filled with $3 \mathrm{ml}$. In the 24 -well 


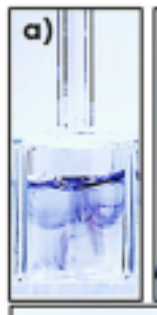

d)

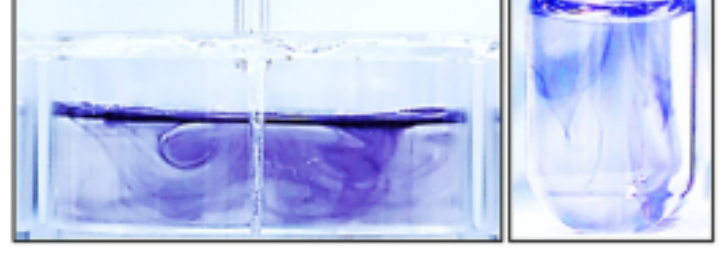

Figure 4. Photos of vortex formation due to plasma action in PBS with $\mathrm{KI}$ and starch reagents for different vessels: (a) 96 well plate, (b) 24 well plate, (c) 12 well plate, (d) 6 well plate, (e) test tube, after $\sim 60$ s from treatment start.

plate and the custom vessel $1.7 \mathrm{ml}$ and $2 \mathrm{ml}$ were introduced to grant the same liquid height $(8 \mathrm{~mm})$ in the two containers. Fresh (untreated) PBS was inserted into the vessel before each test.

Visualization of helium and liquid flows was performed on a Z-type Schlieren [27] optical test bench, equipped with a 3 W green LED light source, a set of parabolic mirrors, a knife-edge mounted vertically on a precision translation and a high frame rate camera (IDT-Streal XS-3, shutter speed: $1 / 10000 \mathrm{~s}$, fps: 60) [28].

The overall plasma-produced oxidizing species distribution in the solution was detected by KI-starch reaction [29]. KI-starch reagents were mixed into the liquid target prior to plasma exposure $\left(0.3 \%_{\mathrm{w}} \mathrm{KI}\right.$ and $0.5 \%_{\mathrm{w}}$ starch).

Non-invasive temperature measurements were performed by means of an infrared camera (Fluke Ti 480 by Fluke) with a $640 \times 640$ pixels detector, an accuracy of $\pm 2{ }^{\circ} \mathrm{C}$ and a resolution of $0.1{ }^{\circ} \mathrm{C}$. The initial calibration of the camera was done using an optical probe used also for gas temperature measurements (Fotemp, Optocon). The emissivity was corrected with the calibration procedure. The camera was mounted so to have the infrared sensor parallel to the sample surface and positioned $10 \mathrm{~cm}$ above it. Due to the intense absorption of water in the infrared spectrum due to the fundamental $\mathrm{O}-\mathrm{H}$ stretching vibrations, the infrared acquisition is axpacted to be mediated over a layer of $50 \mu \mathrm{m}$ under the liquid surface [30].

The mass loss $(\Delta m)$ due to evaporation was evaluated through a high sensibility balance (MettlerToledo ME403). Videos were acquired with a Canon EOS 6D equipped with a $100 \mathrm{~mm}$ macro lens (Canon series $4 L$ ). The recording were effectuated with a frame rate of $30 \mathrm{fps}$ and a shutter time of $1 / 50 \mathrm{~s}$.

\section{Results}

The pictures in figure 4 show that the modification of the fluid

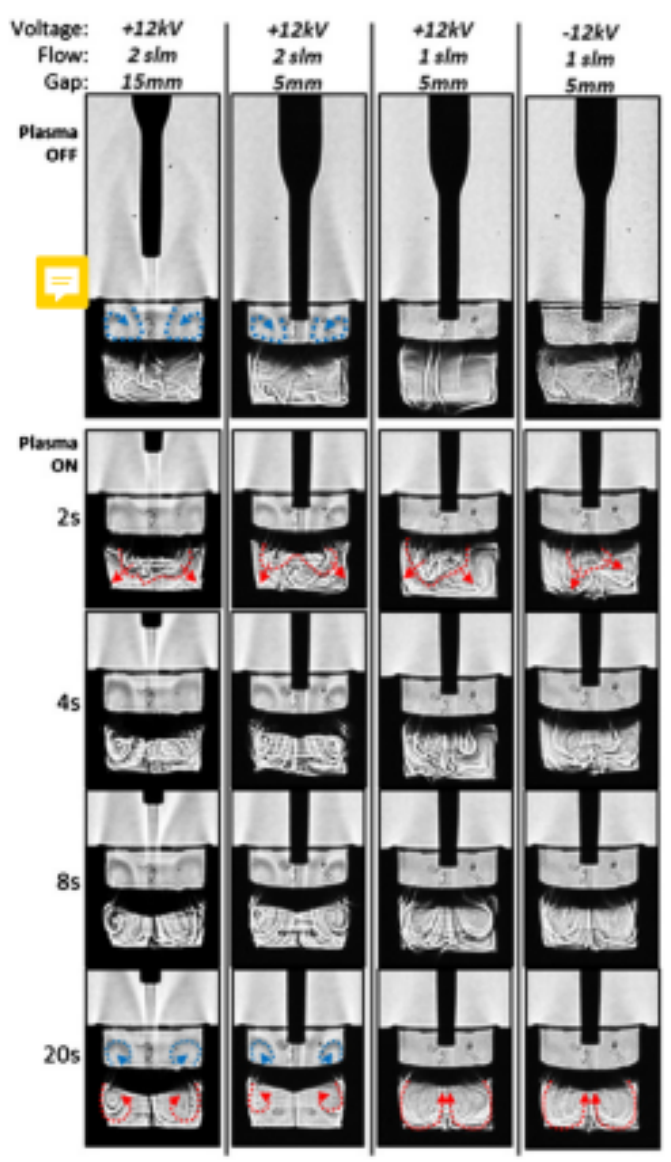

Figure 5. Schlieren acquisitions. Superimposed blue arrow are to help the visualization of vortexes in the gas phase while red lines/arrows are used to highlight turbulent fronts and vortexes in the liquid phase.

dynamic regime in the liquid phase can take place in vessels $(96,24,12$ and 6 well plates as well as in glass test tubes) filled with volumes and liquid typical of biomedical studies.

This direct comparison between different plates clearly demonstrates that the phenomenon is not vessel dependent and can potentially take place in very small volumes down to few liundacals of $\mu$. The plicuonucuva is cleanly uvt specific to a particular vessel geometry but can affect potentially any container commonly adopted in biomedicine. In all investigated cases, the PBS containing KI and starch reagents was treated with the PG ( $+12 \mathrm{kV}_{\text {peak }}, 1 \mathrm{kHz}, 1 \mathrm{slm}, 5 \mathrm{~mm}$ gap).

For all vessels, there is a clear accumulation of reactive species at the surface, that appears of darker color, and the formation of steady state vortexes is observed after few tens of seconds after plasma ignition. The vortex flow direction is the same in all cases: radially outward along the liquid surface and upward at the center of the vessel (i.e. clockwise stream on the right-hand side of the vessels).

The wortexes are the 9 D pmiection on the camera plane of focus of an actually 3D vortex structure with toroidal shape and centered on the jet axis. 
Considering that the characteristic length scale of the observed flow is of the order of one centimeter and the rotation of the vortex in the order of seconds, a velocity of $\sim 1 \mathrm{~cm} \mathrm{~s}^{-1}$ is deduced.

Knowing that the diffusion coefficients for many reactive species in the liquid phase is relatively low $\left(D=\sim 10^{-5} \mathrm{~cm}^{2} \mathrm{~s}^{-1}\right)$ and assuming a mass fraction (c) of 1 at the surface and 0 at the bottom of the well is possible to estimate a mass diffusion flux $(J)$ in the order of $\sim 10^{-5} \mathrm{~g} \mathrm{~cm}^{-2} \mathrm{~s}^{-1}$ according to equation (1) [31]:

$$
J=-\rho D \nabla c
$$

Where $\rho$ is the liquid density assumed in this case to be the same as water $\left(1 \mathrm{~g} \mathrm{~cm}^{-3}\right)$. The mass flux can be expressed also as follow:

$$
J=\rho u u_{\mathrm{D}}
$$

Where $u_{\mathrm{D}}$ is the velocity of diffusion of the reactive species generated at the surface. In this case the diffusion velocity is in the order of $10^{-5} \mathrm{~cm} \mathrm{~s}^{-1}$.

The comparison of the diffusion velocity with the one of the vortexes (several order of magnitude higher) suggests that the convective mixing plays a dominant role in the transport of chemical species in the bulk liquid in the investigated case [20. 32]. This leads to a non-uniform distribution but also to a relatively fast transport from the surface to the bottom of the vessel in the well plates. As later demonstrated, the vortex formation is not determined by the circular base of the vessels. Leveraging on this aspect and to allow the implementation of Schlieren imaging, the following results were collected on the custom vessel with the same characteristic length of the 24 well plate but with a square base. A selection of frames extracted from Schlieren acquisitions is presented in figure 5. The horizontal shadow visible in the middle of the well is casted by the meniscus formed at the surface of the liquid in proximity of the glass wall

As visible in the first row of figure 5, for none of the considered cases the helium flow alone (plasma off) was able to induce the formation of a stable regime in the liquid sample even after several tens of minutes. The helium flow is characterized by a Reynolds number of 150 and 290 at 1 and $2 \mathrm{slm}$ of gas flow. As visible this gives a laminar flow at the outlet of the nozzle [33]. Random fluctuation in the liquid density appear due to the dry helium cooling effect because of water evaporation, but no steady state regime is achieved in this condition

For the cases at higher helium flow (figure 5 first row, two left columns) we observe that stable vortexes are generated in the gas above the liquid surface.

Moreover, a column of helium moving upward is clearly visible to enclose the source capillary reducing the mixing with ambient air, in agreement with the numerical simulation reported by Mohades et al for a similar configuration [34].

Following the plasma ignition, a fast moving front is initiated, propagating from the impinging point and moving away from it in the depth of the liquid, is observed as depicted in figure 5 'plasma on $2 \mathrm{~s}$ ' for every experimental conditions. This fast moving front, typieal of the first few sesonds, ean

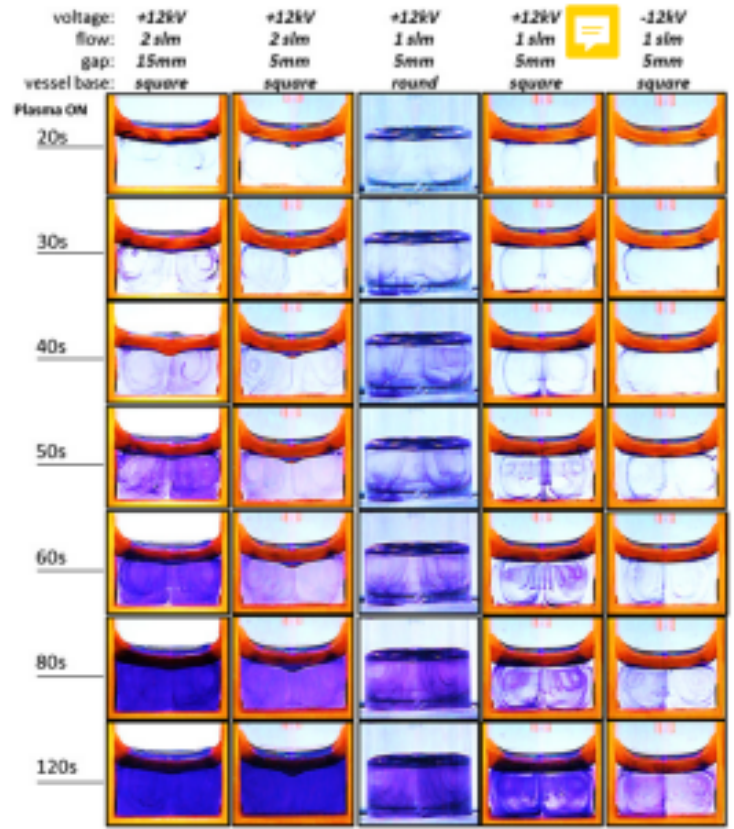

Figure 6. Temporal evolution of target solution containing KI-starch reagents during plasma exposure for different operating conditions.

be associated with the fast flow already reported in details by the group of Kawasaki [12-14, 17].

As hypothesised in one of their work [14] this fast flow, taking place in the first few seconds after plasma ignition, could be hindered by vortex recirculation moving in the opposite direction (upward under the impinging point).

This hypothesis is here confirmed through Schlieren in the transition visible in figure 5 rows $4 \mathrm{~s}$ and $8 \mathrm{~s}$. From the fast liquid flow moving sway from the impinging point the liquid velocity field evolves into a stable toroidal vortex recirculation. The vortex stability was verified for up to several minutes after plasma ignition and presenting no significant variations over this period. These vortexes appear to rotate faster and have smaller dimensions for the cases at higher flow rate $(2 \mathrm{slm})$ nearly mirroring the vortexes formed in the gas phase (see figure 5 row $20 \mathrm{~s}$ ).

The cases at lower flow rate $(1 \mathrm{slm})$ induce wider vortexes always flowing in the same direction. No significant influence of the voltage pulse polarity is ohserved on the fluid dynamic induced inside the liquid (figure 5 third and right-hand side columns).

In figure 6 are presented the results achieved from the addition of KI-starch reagent in the liquid. The cases are the same as those investigated with Schlieren technique. The only exception is the central column that show frame obtained on a standard round well. KI starch distributions for different volumes, vessels and treatment times but typical of most of plasma jet in vitro experiments, as documented in figure 6 , is probably the most striking evidence that reactive species transport in gas and liquid phase has to be in detail considered. At a first glanea, one indeed unambiguously observe! that very 


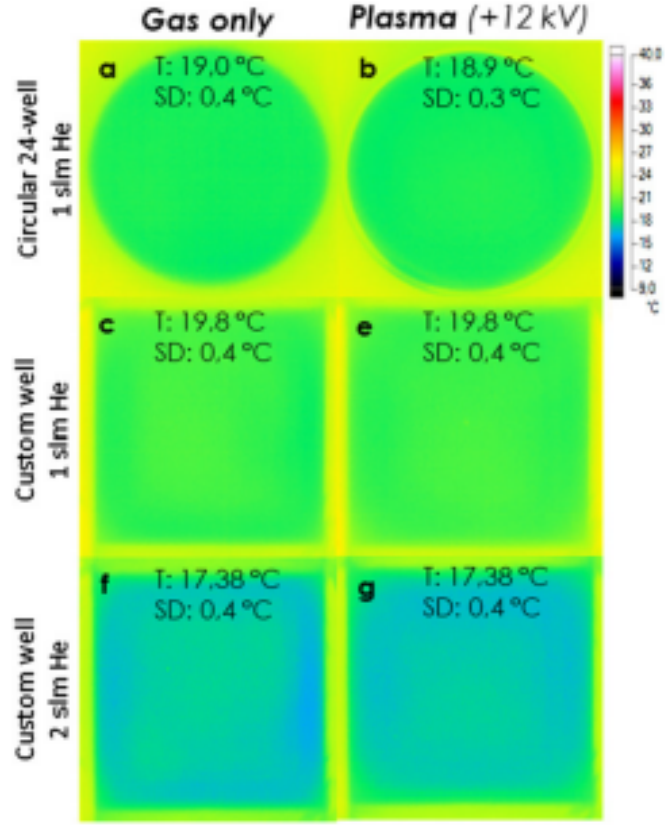

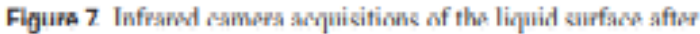
$80 \mathrm{~s}$ of exposure. The gap distance was $5 \mathrm{~mm}$ for all cases. The cases (a), (c) and (f) are without plasma and (b), (e) and (g) with plasma ( $12 \mathrm{kV}$ and $1 \mathrm{kHz}$ ).

different scenario for reactive species delivery are measured and will play a critical role in any biological assays on plasma treatment. As expressed in the introduction, this is the main motivation of this paper, and is probably to be accounted for in plenty of in vitro experiments with other setups, plasma sources and operating conditions.

For all considered cases, in the first $20 \mathrm{~s}$ after the plasma ignition, it is observed an accumulation of reactive species at the surface of the liquid. These species are transported into the bulk of the liquid starting from $30 \mathrm{~s}$ after the start of the plasma.

The path of the oxidized reagents follows the vortex structures previously identified through Schlieren imaging. For the case at higher flow rate the vortex are faster and lead to a nearly uniform diffusion of the plasma induced species within $80 \mathrm{~s}$. The cases at lower flowrate are characterized by larger and slower vortexes which induce reactive species flow closer to the vessel wall and a central column in correspondence of the impinging primt. The volume of liquid at die centue of diese vortexes forms a torus of clear, unreacted, liquid up to $80 \mathrm{~s}$ after the start of the plasma for some cases. The oxidation of increasingly more reagents and their diffusion makes gradually harder to visualize the vortexes after times longer than $60 \mathrm{~s}$.

A selection of IR camera acquisitions is reported in figure 7 . Both the pictures and the extracted temperature values show how the heat transfer is strongly dependant on the gas flow rate and nearly unaffected by the presence of the plasma or the shape of the vessel. The images also show a nearly uniform temperature over the liquid surface with a very low deviation between the various points.

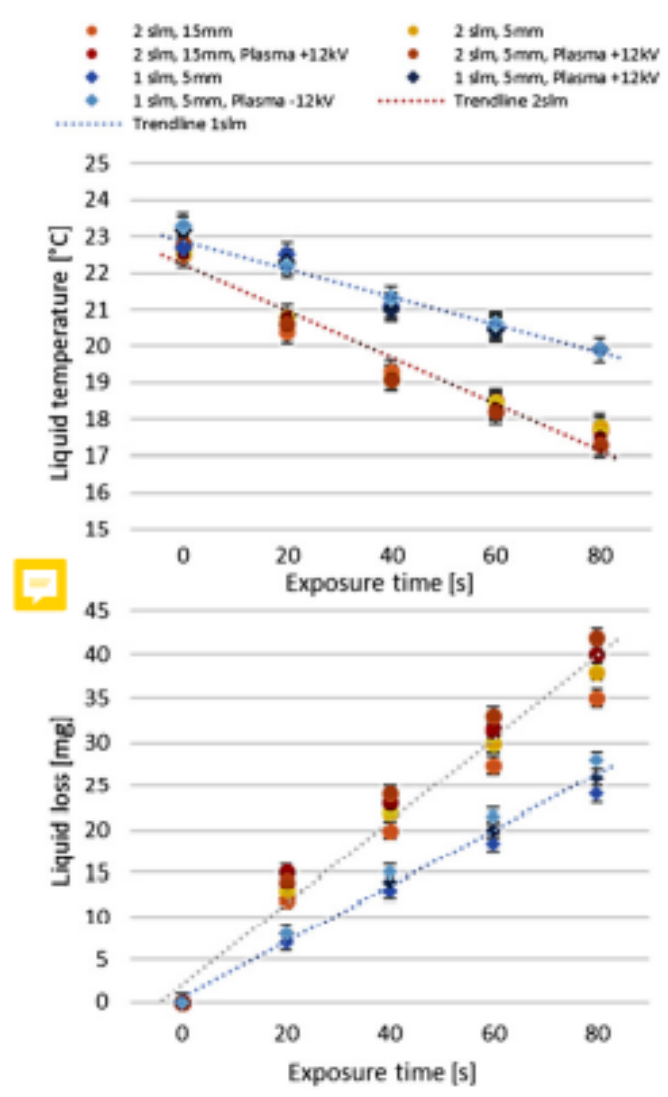

Figure 8. Liquid surface temperature (up) and liquid loss (bottom) over treatment time.

The average temperatures of the liquid surface for different conditions are reported in figure 8. A general decrease of the temperature over time is reported for all cases. Also there is a clear grouping of the experimental points along two different trend lines, one characteristic of each investigated gas flow. With $1 \mathrm{sm}$ of He gas flow it is recorded an average loss of $0.036^{\circ} \mathrm{C} \mathrm{s}^{-1}$ while at 2 shan the luss is of $0.065^{\circ} \mathrm{C} \mathrm{s}^{-1}$.

Similar trends are also observed for the liquid mass loss recorded during the treatments. The cases divides into two groups according to the He flow value. An average evaporation of $\left.177 \mathrm{~g}^{-1} \times \mathrm{s}^{-1} \times \mathrm{m}^{-2}\right)$ and $\left.180 \mathrm{~g}^{-1} \times \mathrm{s}^{-1} \times \mathrm{m}^{-2}\right)$ are recorrled for the $1 \mathrm{slm}$ and $2 \mathrm{slm}$ conditions respectively. No significant difference between the gas only and the plasma treatment is observed also in this case, probably in virtue of the very low power characteristic of the adopted plasma source. In addition, the polarity of the excitation pulse does not seems to play a key role for the measured phenomena in thiy experimental conditions.

\section{Discussion}

In this section, the effects possibly causing the vortex formation are addressed one by one with reference to the adopted 
experimental setup and supported or discarded thanks to experimental evidences or theoretical considerations. The analysis is performed for the square custom vessel who has the advantage of allowing Schlieren imaging visualization (figure 5).

\subsection{Buoyancy forces and Marangoni effect}

One of the most common cause of flows in fluids are density gradients caused by non-uniform heat transfer (i.e. convection). The Richardson number ( $\mathrm{Ri}$ ) is usually adopted to express the ratio of a buoyancy term over a flow shear term and often used to evaluate the importance of natural convection over forced convection [35]. Typically, the natural convection is negligible when $\mathrm{Ri}<0.1$ and forced convection is negligible for $\mathrm{Ri}>10$. Neither is negligible when $0.1<\mathrm{Ri}<10$. It is simplest to express Ri using a combination of the Grashof number (Gr) and the Reynolds number ( $\mathrm{Re})$ :

$$
\begin{gathered}
\mathrm{Ri}=\frac{\mathrm{Gr}}{\mathrm{Re}^{2}} \\
\mathrm{Gr}=\frac{g \beta\left(T_{\mathrm{s}}-T_{\infty}\right) L^{3}}{\nu^{2}} \\
\operatorname{Re}=\frac{u L}{\nu}
\end{gathered}
$$

Where $u$ is the flow speed $\left(\mathrm{m} \mathrm{s}^{-1}\right), L$ the characteristic length $(\mathrm{m})$, in this case the vessel side $(16 \mathrm{~mm}), \nu$ the kinematic viscosity $\left(\mathrm{m}^{2} \mathrm{~s}^{-1}\right), g$ the gravity acceleration $\left(9.8 \mathrm{~m} \mathrm{~s}^{-1}\right), \beta$ is the coefficient of thermal expansion $\left(\mathrm{K}^{-1}\right), T_{\mathrm{s}}$ and $\mathrm{T}_{\infty}$ the temperature of the surface and of the unperturbed fluid $(K)$. Being the initial liquid velocity zero $(u=0)$, no forced convection, the $\mathrm{Re} \& 1$ and therefore $\mathrm{Ri} \gg 1$. With this assumption, the buoyancy forces in the liquid phase should be not negligible in our case. The same analysis on the He flow $\left(u=12.6 \mathrm{~m} \mathrm{~s}^{-1}\right.$, $L=1.6 \times 10^{-2} \mathrm{~m}, \nu=1.12 \times 10^{-2} \mathrm{~m}^{2} \mathrm{~s}^{-1}, \beta=3.37 \times$ $10^{-3} \mathrm{~K}^{-1}, T_{\mathrm{s}}=74^{\circ} \mathrm{C}, \mathrm{T}_{\infty}=17^{\circ} \mathrm{C}$ gives a $\mathrm{Ri}=77 \times$ $10^{-5}$ which suggest a negligible impact of buoyancy on the gas phase as also confirmed by the laminar downward column of He visible in Schlieren acquisitions.

In the investigated setup the only two possible sources of thermal effects on the liquid are the heating due to the plasma discharge and the cooling due to evaporation as a consequence of the helium flow (initially very low humidity content). In light of the short treatment time and the initial thermal equilibrium, the heat transfer across the well walls is considered negligible. The variation of the mean liquid surface temperature, recorded with the IR camera (figure 7), shows a predominant cooling effect in all cases. Moreover, it is measured that the surface temperature is essentially affected by the gas flow with little or no impact of other parameters such as gap distance or the presence of the plasma. This trend is supported in the graphs reporting the temperature variation and the mass loss due to evaporation (figure 8).

Considering the gas only cases and assuming the enthalpy of vaporization of PBS to be equal to that of water, $2257 \mathrm{~J} \mathrm{~g}^{-1}$ $\left(H_{\mathrm{v}}\right)$, for a mean mass loss due to evaporation over $80 \mathrm{~s}(\Delta \mathrm{m})$ of 24 and $36.5 \mathrm{mg}$ for 1 and $2 \mathrm{slm}$ respectively, it is possible, through the following equation, to estimate a mean cooling power of $0.68 \mathrm{~W}$ and $1.03 \mathrm{~W}$.

$$
P_{\text {evap }}=\frac{\Delta m * H_{v}}{t}
$$

The plasma source adopted in this setup is characterized by a particularly low power consumption. The power delivered to the plasma reactor from the pulse generator vary according to the operating conditions but for the considered cases it is was measured to be always in the range of $0.2-0.3 \mathrm{~W}$ [36]. Additionally, only a fraction of this power is expected to be converted into heat while most goes into the excitation of helium atoms and other species. This is also confirmed by simple temperature measurement performed on the gas flow on the capillary axis $1 \mathrm{~mm}$ under the nozzle (figure 1 position ' $\mathrm{a}$ ') and at the same height but shifted $5 \mathrm{~mm}$ radially (figure 1 position 'b') where the helium flow exit the well moving upward. The gas at the exit of the capillary was recorded to be between 21 and $22{ }^{\circ} \mathrm{C}$ and to increase at $24.6^{\circ} \mathrm{C}$ when the plasma was present. The gas is then cooled by the water evaporation reaching temperatures in the range of $17^{\circ} \mathrm{C}-19^{\circ} \mathrm{C}$ when exiting the well.

The difference between the cooling and heating powers suggest that any possible thermal effect is mainly controlled by the helium gas induced evaporation. Since this effect is also present in the gas only cases where the vortexes are not observed this analysis supports that vortexes formation is very likely independent of the thermal balance between gas flow cooling and liquid evaporation.

Furthermore, as reported by Kovacevic et al [18] for a close setup but using Ar gas and a larger vessel, the gas only cooling effect is expected to cause liquid rotation opposite to the one here reported. Anyway, this effect is not observed in the present case on smaller vessels representative of those commonly adopted in biomedical $i n$ vitro experiments. While in Kovacevic experiment the liquid cooled by the gas was in contact and possibly heated by the surrounding liquid at ambient temperature, in this case the cooled liquid is contoured by thermally insulating walls. Moreover, the lower liquid depth could also play a role in the observed differences. Also in the present case, the smaller surface is completely covered by the He flow causing a uniform evaporation and not presenting surface temperature gradients that may induce liquid flows. This consideration leads to another possible cause of liquid flow due to temperature gradients: Marangoni stresses [37, 38]. These stresses could be induced by gradients in the surface tension leading to flows from regions of high to low surface tension. The uniform temperature distribution at the surface observed with the infrared camera allows us to exclude possible flow duc to surface unequal heating/cooling. As presented in details in the works by Thagard et al $[19,25]$ Marangoni stresses can be caused by concentration gradients of surfactants (e.g. PFOA, Rhodamine B, etc). The degradation of this chemical compound by the plasma discharge can impose a gradient sufficient to induce a liquid motion near the surface.

The absence of surfactants in our liquid target considerably reduces the possibility of such effect. 
We conclude that effects other than thermal ones must cause the vortex formation.

\subsection{Gas flow shear stress}

Another possible origin of the vortexes could be the gas flow impinging on the liquid surface and propagating radially across its surface. The motion of the gas could transfer momentum to the liquid by shear stress on its surface and initiate a radial liquid motion from the center to the border of the well (figure 1). The condition in which a column of gas impinges on a liquid surface is variously addressed in non-plasma literature $[39,40]$. It is accepted that the energy transferred from the gas stream appears in the liquid in three distinguished forms: displacement of the liquid (gravitational energy), motion of the liquid (kinetic energy) and increase of the surface area due to the dimple formation instead of a flat surface (surface energy). For this discussion it will be disregarded the effect on the liquid displacement and the surface energy which usually cause the formation of a dimple under the gas injection nozzle and that in this configuration is observed only for the cases at higher flow rate (figure 6, two first columns on the left) $[9,21,41]$. These phenomena are not associated with modification of the flow in the bulk liquid and therefore will not be considered in this discussion.

The transfer of kinetic energy from the gas flow impinging on the liquid and then flowing across its surface can take place through shear stress and can cause vortex formation with characteristics similar to the one here observed $[40,42]$. Therefore, vortex formation similar to the one observed could be possibly caused by the gas flow independently from the presence of plasma. Following the reasoning presented by Semenov et al [20] it is possible to express the shear stress at the gas-liquid interface $\left(\tau_{i}\right)$ in terms of the gas flow velocity $\left(u_{\varepsilon}\right)$ according to the following formula:

$$
\tau_{\mathrm{i}}=\frac{\rho_{\mathrm{g}} \nu_{\mathrm{g}} u_{g}}{\Lambda_{g}}
$$

Where $\Delta_{g}$ is the thickness of the viscous sublayer that is expressed as:

$$
\Delta_{g}=\sqrt{\frac{\nu_{g} L}{u_{g}}}
$$

Where $u_{\bar{g}}$ is the gas speed (m s ${ }^{1}$ ), $L$ the characteristic length $(\mathrm{m}), \nu_{\mathrm{g}}$ the kinematic viscosity $\left(\mathrm{m}^{2} \mathrm{~s}^{-1}\right), \rho_{\mathrm{g}}$ the gas density $\left(\mathrm{kg} \mathrm{m}^{-3}\right)$. In virtue of the laminar flow and the very low Ri number, it is reasonable to assume as a first approximation that the speed of the gas over the liquid surface nearby the impinging point is close to the speed at the exit of the capillary $\left(12.5 \mathrm{~m} \mathrm{~s}^{-1}\right.$ for $1 \mathrm{slm}, 25.1 \mathrm{~m} \mathrm{~s}^{-1}$ for $\left.2 \mathrm{slm}\right)$. The density and viscosity are easily found in the literature $\left(\rho_{\mathrm{g}}=0.1785 \mathrm{~kg} \mathrm{~m}^{-3}\right.$, $\left.\nu_{g}=1.12 \times 10^{-2} \mathrm{~m}^{2} \mathrm{~s}^{-1}\right)$ while as characteristic length is again chosen the side of the vessel $\left(I=16 \times 10^{-2} \mathrm{~m}\right)$ The shear stress is in this way estimated to be $0.67 \mathrm{~Pa}$ and $1.87 \mathrm{~Pa}$ for 1 and $2 \mathrm{~s} \mathrm{sm}$ He flow rate. These values are comparable with those reported by Semenov et al [20].

Moving further it is possible to use equation (9) to try to have an estimate of the expected liquid velocity close to the surface due to the shear stress imposed by the He flow.

$$
u_{1}=\tau_{\mathrm{i}}^{\mathbf{Y}}\left(\frac{L}{\rho_{1} \mu_{1}}\right)^{\frac{1}{3}}
$$

The resulting velocity is in the order of $10-60 \mathrm{~cm} \mathrm{~s}^{-1}$ which is comparable with other values reported in the literature but probably higher than the real one due to the initial approximation on the gas velocity $[20,43]$.

Nevertheless, for the investigated setup, for none of all cases with only gas flow (figure 5, first row) it is observed the formation of vortexes in the liquid even after several minutes. For the same gas flow condition vortexes are instead observed to form within few seconds after that the plasma is ignited. An hypothesis that could be advanced is that the gas flow only induced liquid motion may be hindered by the liquid viscosity and inertia as well as the thermal effect possibly favouring a motion with opposite direction (see previous section). These contrasting effects could potentially explain the instability observed in the Schlieren acquisitions for the gas only cases and the impossibility for a stable regime to take place (figurc 5, first row).

As suggested in various papers, the plasma discharge can possibly increase the gas flow velocity due to thermal expansion as a consequence of the high temperature reached in proximity to the electrode. Gas velocity alteration due to plasma induced gas heating is expected to be in the order of $10 \%-30 \%$ according to the literature on similar plasma configurations $[20,21,44]$. In this study the present hypothesis was investigated comparing the case with gas only at $1 \mathrm{slm}$, which present no liquid circulation, with the results achieved at $1 \mathrm{slm}$ with plasma (vortex) and 2 slm for the gas only (no vortex). If the plasma effect was only of inducing a gas yelocity increase the vortex formation would have been obtained also with an increase of $100 \%$ of the flow rate of the gas only. The evidence that this is not the case supports the hypothesis that the vortex formation is not just a consequence of a plasma induced temporary increase of the gas mean velocity.

Moreover, the gas vortexes above the surface remain similar with and without plasma suggesting a limited alteration of the gas velocity. Similar observation were reported by Hefny et al [23] for a similar plasma setup, helium flow and gap distance. The gas velocity increase alone is therefore not the cause of the observed liquid circulation.

Anyway, plasma can also induce alteration of the gas flow velocity profile potentially leading the gas flow from being purely laminar to presenting tangential velocity components (swirl). In the investigated case a tangential component on the shear stress at the liquid surface could explain the formation of vortexes such as those observed. Examples of such flows are well-known for example to take place in liquid near a rotating disk or in cylindrical cavity with rotating top lid [45]. Moreover, the formation of such vortexes has heren confirmed to be strongly dependent on tangential components also in the numerieal simulation of an APPJ impinging on a liquid surfaces [20]. Furthermore it is documented how axisymmetric liquid flows are usually unstable with respect to tangential perturbations and that plasma discharge generation can induce 
perturbation within the jet shear layer $[46,47]$. This explanation is also supported by the observed dependency between gas flow and vortexes dimension (figure 5). The higher gas flow (2 slm) leads to higher gas velocity and shear stress on the liquid surface which generates faster and smaller vortexes but only if there is a plasma-induced swirl component to trigger the process. Therefore, the plasma-induced gas flow modification, in terms of introduction of a tangential component, is possibly at the origin of the observed vortex formation.

\subsection{Electrohydrodynamic $(E H D)$ forces}

Another possible cause for the vortex are EHD forces generated by the plasma ionization wave (IW) impinging and propagating along the liquid surface and the charging of the surface itself. Darny et al [28] reported on the non-negligible effect of EHD forces acting on the He flow of the same APPJ investigated in this work while impinging on a solid target. They demonstrated the critical role played by negative ions in inducing modification of the gas propagation. In the present case the ionic wind is probably of less importance, even negligible, in the building of a stable $\mathrm{He}$ channel connecting the APPJ and the target in virtue of the higher gas flow, smaller gap and smaller orifice compared with the work of Darny (0.5 slm, $20 \mathrm{~mm}$ gap and $4 \mathrm{~mm}$ diameter). Van Doremaele et al [48] in their work on a helium APPJs interacting with solid dielectric target highlighted the dominant role played by deposited surface charges in governing the EHD effects in the gas phase. It must be remarked anyway that PBS, used here as a target, is characterized by a relatively high conductivity $\left(\sim 1.2 \mathrm{~ms} \mathrm{~cm}^{-1}\right)$. Compared to a solid dielectric substrate where deposited charges can stray for several seronds [49], on the PBS surface they are expected to have a relatively short residence time leading to a faster charge dissipation.

The ionic wind, here a more proper expression would be 'ionic flow', that is of the most interest in our case is the one produced inside the liquid. The IW striking and propagating over the surface and the charge transportation through the formed conductive channel lead to the charging of the liquid surface. This in turn induces an electric field that can have values up to $100 \mathrm{kV} \mathrm{cm}^{-1}$ as reported in a numerical investi gation by Norberg et al for a configuration similar to the one here discussed [50].

This high electric field, even if effeetive-enly-for a short pcriod cqual to the applicd voltage pulsc $(4 \mu \mathrm{s})$ or the deposited surface charge residence time, may be enough to put into motion the liquid closer to the surface. This hypothesis is also supported by the work of Kanazawa et al [51], on pulsed streamer discharge (without gas flow) generated over a water solution can induce the formation of vortexes in the liquid. This was also observed with Schlieren for a similar configuration over silicon oil by Ohyama et al [52] where the phenomenon was linked to a ionic wind and liquid interface interaction and evaluated by means of a dimensionless index, the conductive EHD Rayleigh number (Ehd), defined as follow:

$$
\text { Ehd }=\frac{I L^{3}}{\rho_{g} \nu_{g}^{2} \mu_{i} A}
$$

Where $I$ is the current, $L$ the characteristic length (gap distance), $\rho_{g}$ the gas density, $\nu_{g}$ the kinematic viscosity of the gas, $\mu_{\mathrm{i}}$ the ion mobility in the gas and $A$ the electrode area. Reliable values for $\mu_{\mathrm{i}}$ can be extracted from the literature $\left(500 \mathrm{~m}^{2} \mathrm{~V}^{-1} \mathrm{~s}^{-1}\right)[53,54]$. The rms value of the current $(I)$ during the voltage pulse vary slightly among the investigated cases and it is always around a mean value of $20 \mathrm{~mA}$. The area $A$ is assumed equal to the area covered by the plasma on the liquid surface (assessed by photo acquisitions to be $16 \mathrm{~mm}^{2}$ ). The Ehd number for our conditions at $5 \mathrm{~mm}$ gap is 141 while for the $15 \mathrm{~mm}$ case is 3800 .

These values are comparable with the range reported by Ohyama ( $\sim 1000$ ), supporting the idea that the observed vortex formation may have the same cause, EHD forces.

In the present work it is possible to speculate on the effect of the excitation voltage polarity. The achieved results show a vortex formation dynamic that is essentially identical for the two polarities probably due to the greater gas inertia compared to possible ionic wind effects (figure 5, two column on the right). The KI-starch investigation instead shows a faster distribution of the reactive species for the positive polarity. This may be attributed to a greater production of reactive species rather than a more effective momentum transfer of the negative polarity. While gaseous negative ions have been proven to be more effective in the transfer of momentum in the gas phase this is not necessarily true for the liquid phase. Anyway it cannot be excluded that polarity, as well as bipolar excitation, may still play an effect in other configurations different from the one here addressed.

\section{Conclusion}

Vessel shape and volume, and induced liquid mixing are critical to APPJ applications to liquid systems as surface and liquid transport can have a critical impact on the reaction kinetics $[9,24]$. The setup configuration in which an APPJ impinges on a liquid surface is widely adopted in plasma medicine.

It is shown how the impinging of an APPJ can induce liquid recirculation also in vessels and liquids commonly adopted for biomedical in vitro studies.

The generated vortexes strongly affect the distribution of long-lived reactive species inside the liquid, leading to nonuniform distributions (e.g. pristine volume of liquid inside the vortexes) but also to a relatively fast transport from the surface to the bottom of the vessel.

This effect can significantly alter the transport and distribution of long-lived reactive species even in volumes as small as few hundreds $\mu \mathrm{l}$ and in turn affect the treatment outcome. Thinking about in vitro cells it is evident how this effect could lead to great variation in the cells exposure to RONS with cells that could potentially be untouched (e.g. inside the vortexes) and other overexposed (e.g. near the surface). Similarly, it must be taken into account that cells might be exposed to RONS concentration several order of magnitude higher/lower than the mean value usually measured over the entire liquid volume.

Especially comparison between plasma treatment and various controls that may include exposure to gas flow only or to liquid solutions with various concentrations of reactive species 
mimicking those produced by the plasma, should take this mixing dynamic into account. These evidences strongly suggest that reactive species transport in gas and liquid phase has to be in detail considered and accounted for in plenty of in vitro experiments even with other setups, plasma sources and operating conditions.

The phenomena at the origin of the vortex formation have been investigated and discussed leading to the final observation that the main causes are EHD forces and gas flow tangential components.

Conversely, the cooling due to evaporation that can significantly reduce the sample temperature is discarded as a possible cause for the liquid motion. Nevertheless, it must be noticed that for longer exposure times (e.g. several minutes) evaporation can surely lead to a decrease in the liquid eight and consequently affect the liquid flow regime.

According to the theoretical considerations here reported, the liquid viscosity as well as the applied voltage are supposed to be key parameters for the observation of this phenomenon. Likewise, the same effects are expected to happen when using other gases such as Ar. Nevertheless, the different gas properties and the resulting different gas flow regimes (e.g. Ar presents much higher $\mathrm{Re}$ numbers compared to $\mathrm{He}$ for same gesmetrieal esntigurations and gas flows) recommend eaution in any possible generalization or direct comparison.

These findings highlight how special attention should be paid to the characterization of sctups adopted for plasma in vitro biomedical experiments. Only diagnostic performed in realistic conditions can give an insight of what is happening during plasma applications.

Future studies will focus on the investigation of a wider range of operating conditions, including different liquid volumes and characteristics (i.e. conductivity and viscosity).

\section{Acknowledgments}

PLASCANCER project INCa-PlanCancer-n ${ }^{\circ} 17 \mathrm{CP} 087-00$ and GdR 2025 HAPPYBIO.

\section{ORCID iDs}

Augusto Stancampiano (1) https://orcid.org/0000-0002-75341235

Eric Robert $\odot$ https://orcid.org/0000-0002-7894-7080

\section{References}

[1] Ji L, Yan W, Xia Y and Liu D 2018 J. Appl. Phys, 123183302

[2] Mussard M D V S, Foucher E, Rousseau A, Search H, Journals C, Contact A, lopscience M and Address I P 2015 J. Phys. D. Appl. Phys. 424003424003

[3] Pouvesle J-M 2019 ISPC 24 (Naples 9-14 June)

[4] Riès D, Dilecce G, Robert E, Ambrico P F, Dozias S and Pouvesle J-M 2014 J. Phys. D: Appl. Phys. 47275401

[5] Stancampiano A, Chung T-H, Dozias S, Pouvesle J-M, Mir L M and Robert E 2019 IEEE Trans. Radiat. Plasma Med. Sci. 4 $335-42$
[6] Kaneko T et al 2015 Biointerphases 10029521

[7] Zhou C, He T and Xia W 2019 Phys. Fluids 26053507

[8] Sasaki S, Honda R. Hokari Y, Takashima K, Kanzaki M and Kaneko T 2016 J. Phys. D: Appl. Phys. 49334002

[9] Taghvaei H, Kondeti V S S K and Bruggeman P J 2019 Plasma Chem. Plasma Process. 39729

[10] Lindsay S M and Yin J 2016 AIChE J. 62222 ?

[11] Kondeti V S S K and Bruggeman P J 2020 J. Phys. D Appl. Phys. areepted

[12] Kawasaki T, Kusumegi S, Kudo A, Sakanoshita T, Tsurumaru T, Sato A, Uchida G, Koga K and Shiratani M 2016 J. Appl. Phys. 11917330 !

[13] Kawasaki T, Kusumegi S, Kudo A, Sakanoshita T, Tsurumaru T and Sato A 2016 IEEE Trans. Plasma Sci. 44 3223-9

[14] Mitsugi F, Kusumegi S, Nishida K and Kawasaki T 2020 IEEE Trans. Plasma Sci. 44 1-6

[15] Rumbach P, Griggs N, Sankaran R M and Go D B 2014 IEEE Trans. Plasma Sci. $\mathbf{4 2} 2610$

[16] Mitsugi F, Kusumegi S, Kawasaki T, Nakamiya T and Sonoda Y 2016 IEEE Trans. Plasma Sci. 44 3077-82

[17] Kawasaki T, Eto W, Hamada M, Wakabayashi Y, Abe Y and Kihara K 2015 Japan. J. Appl. Phys. 54086201

[18] V Kovačević V, Sretenovié G B, Slikboer E, Guaitella O, Sobota A and Kuraica M M 2018 J. Phys. D. Appl. Phys. 51 065202

[19] Thagard S M, Stratton G R, Vasilev M, Conlon P and Bohl D 2018 Plasma Chem. Plasma Process. 38719

[20] Semenov I L, Weltmann K-D and Loffhagen D 2019 J. Phys. D: Appl. Phys. 52315203

[21] Van Rens J F M, Schoof J T, Ummelen F C, Van Vugt D C Bruggeman P J and Van Veldhuizen E M 2014 IEEE Trans. Plasma Sci. 42 2622-3

[22] Shimizu T, Iwafuchi Y, Morfill G E and Sato T 2011 New J. Phys. 13053025

[23] Hefny M M, Pattyn C, Lukes P and Benedikt J 2016 J. Phys. D. Appl. Phys, 49 404002

[24] Brubaker T R, Ishikawa K, Kondo H, Tsutsumi T, Hashizume H, Tanaka H, Knecht S D, Bilén S G and Hori M 2019 J. Phys. D: Appl. Phys. 52075203

[25] Mededovic Thagard S, Stratton G R, Dai F, Bellona C L, Holsen T M, Bohl D G, Paek E and Dickenson E R V 2017 J. Phys. D. Appl. Phys. 50014003

[26] Darny T, Pouvesle J-M, Puech V, Douat C, Dozias S and Robert E 2017 Plasma Sources Sci. Techinol. 26045008

[27] Traldi E, Boselli M, Simoncelli E, Stancampiano A. Gherardi M, Colombo V and Settles G S 2018 EPJ Tech. Instrum.

[28] Darny T, Pouvesle J-M, Fontane J, Joly L, Dozias S and Robert E 2017 Plasma Sources Sci. Technol. 26105001

[29] He T, Liu D, Liu Z, Sui W, Liu Z, Rong M and Kong M G 2018 J. Phys. D Appl. Phys. 419545

[30] Curcio J A and Petty C C 1951 J. Opt. Soc. Am. 41302

[31] Mills A F 1998 Int. J. Heat Mass Thansfer 411955

[32] Bruggeman P J et al 2016 Plasma Sources Sci. Technol. 25 053002

[33] Stancampiano A, Simoncelli E, Boselli M, Colombo V and Gherardi M 2018 Plasma Sources Sci. Techinol. 27125002

[34] Mohades S. Lietz A M, Kruszelnicki J and Kushner M J 2020 Plasma Process. Polym. 171

[35] Kotsovinos N E 1975 A Study of the Entrainment and Turbulence in a Plane Buoyant Jet (California Institute of Technology 
$\mathrm{q}=$ Interfacial stress and convection \& $\mathrm{f}=\mathrm{false}$ (accessed July $16,2020)$

[38] Alexander N, Simanovskii I and Legros J C 1993 Interfacial Comvection in Multilayer Systems (Berlin: Springer)

[39] Rosler R S and Stewart G H 1968 J. Fluid Mech. 31163

[40] Hwang H Y and Irons G A 2012 Metall. Marer. Trans. B 43302

[41] Stancampiano A, Simoncelli E, Boselli M and Colombo V 2018 Plasma Sources Sci. Technol. 27125002

[42] Nguyen A V and Evans G M 2006 Appl. Math. Modelling 30 1472

[43] Verlackt C C W, Van Boxem W and Bogaerts A 2018 Phys. Chem. Chem. Phys. 206845

[44] Reuter S, Von Woedtke T and Weltmann K D 2018 J. Phys. D. Appl. Phys. 51233001

[45] Escudier M P 1984 Exp. Fluids 2189

[46] Moffatt H K and Tsinober A 1992 Annu. Rev. Fluid Mech. 24 281
[47] Morabit Y, Whalley R D, Robert E, Hasan M I and Walsh J L 2020 Plasma Process. Polym. 17 I

[48] Van Doremaele E R W, Kondeti V S S K and Bruggeman P J 2018 Plasma Sources Sci. Technol. 27095006

[49] Wild R, Gerling T. Bussiahn R, Weltmann K D and Stollenwerk L 2014 J. Phys. D. AppL. Phys. 47042001

[50] Norberg S A, Johnsen E and Kushner M J 2015 J. Appl. Phys. 118013301

[51] Kanazawa S, Kawano H, Watanabe S, Furuki T, Akamine S, Ichiki R, Ohkubo T, Kocik M and Mizeraczyk J 2011 Plasma Sources Sci. Technol. 20034010

[52] Ohyama R, Inoue K and Chang J S 2007 J. Phys. D: Appl. Phys. 40573

[53] Viehland L A and Mason E A 1995 At. Data Nucl. Data Tables $6037-95$ 